\title{
Acute kidney injury risk factor recognition in three teaching hospitals in Ethiopia
} \author{
Y Tadesse, ${ }^{3} \mathrm{MD}$; J Williams, ${ }^{1} \mathrm{MD}$; A Phillips, ${ }^{1} \mathrm{MB} \mathrm{ChB}, \mathrm{BSc}, \mathrm{MD}, \mathrm{FRCP}$ \\ ${ }^{1}$ Institute of Nephrology, Cardiff University School of Medicine, Cardiff, Wales, UK \\ ${ }^{2}$ College of Medicine and Health Sciences, University of Gondar, Gondar, Ethiopia \\ ${ }^{3}$ Renal Unit, Tikur Anbessa Hospital, Addis Ababa, Ethiopia
}

L Phillips, ${ }^{1}$ BMed Sci; N Allen, ${ }^{1}$ MB ChB; B Phillips, ${ }^{1}$ medical student; A Abera, ${ }^{2}$ MB ChB; E Diro, ${ }^{2}$ MB ChB, PhD; S Riley, ${ }^{1}$ MB ChB, MD;

Corresponding author: A Phillips (Phillipsao@cf.ac.uk)

Background. A key objective of the Nephrology Sister Centre Programme between the renal units in Cardiff and Addis Ababa, sponsored by the International Society of Nephrology, is to facilitate development of the local clinical service in Ethiopia specifically focused on the management of acute kidney injury (AKI).

Objectives. To examine the relationship between AKI risk factor recognition and monitoring of renal function in three hospitals in Ethiopia. Methods. Cross-sectional data were gathered regarding renal function monitoring, recording the presence of AKI risk-associated comorbidities and prescription of nephrotoxic medications across the disciplines of medicine, surgery, obstetrics and gynaecology.

Results. Patients were more likely to have their renal function checked at the hospital with specialist services. Across all centres, the highest proportion of patients who had renal function measurements were those admitted to a medical ward. There was a positive relationship between documented comorbidities and the measurement of renal function but not between the prescription of nephrotoxic drugs and measurement of renal function.

Conclusion. There was great variability in the extent to which doctors recognised the presence of risk factors for the development of AKI. Failure to identify these risk factors represents a lost opportunity to identify patients at high risk of developing renal injury who would benefit from renal function monitoring.

S Afr Med J 2013;103(6):413-418. DOI:10.7196/SAMJ.6424

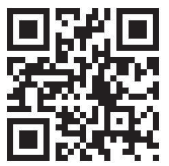

The high morbidity and mortality associated with acute kidney injury (AKI) in Ethiopia represents an important challenge to its health community. A recent initiative sponsored by the International Society of Nephrology's Sister Centre Programme between the renal units of the University Hospital in Cardiff and Tikur Anebassa Hospital in Addis Ababa has the broad goal of significantly raising the profile of renal disease in Ethiopia. The specific objectives are to enhance nephrology education and to facilitate development of the local clinical service, particularly in the management of AKI. Information regarding the current needs and activity, which are the essential tools required for design of a structured plan for service, are currently lacking; the lack of available data for presentation to policymakers is a major obstacle to any plea for investment and service development.

It is known that, in many cases, AKI is reversible and self-limiting with supportive management and temporary support of renal function through dialysis. A study from Sudan demonstrated that, of a cohort of patients with severe acute renal failure, $60 \%$ recovered renal function following appropriate management. ${ }^{[1]}$ However, in developing countries, which lack access to renal replacement therapy, mortality rates remain high. ${ }^{[2-4]}$ Failure of active monitoring of renal function may lead to missed opportunities for the treatment of prerenal AKI which, if identified, may be reversed with appropriate management of fluid and electrolyte disturbances. These challenges are particularly apparent in Ethiopia, where nephrology services for the entire population of 80 million are based exclusively in Addis
Ababa. Even within the specialist centre in Addis Ababa, access to dialysis for patients presenting with acute (or chronic) renal failure is limited, owing to the erratic supply of consumables. However, some patients may be referred to private dialysis facilities, treatment then being limited by the ability of the patient and family to raise the necessary funds. This limitation of access to dialysis highlights the importance of early identification of AKI and reversal of kidney injury, obviating the need for dialysis and, ultimately, reducing the drain on the limited resources.

Such a strategy of targeted monitoring relies on the ability to detect patients with risk factors for developing AKI. One important predisposing factor is the presence of pre-existing chronic renal impairment, with acute-on-chronic AKI thought to represent up to $40 \%$ of all cases of AKI. ${ }^{[5]}$ Importantly, any episode of superimposed dialysis-requiring AKI in patients with chronic kidney disease (CKD) has been shown to be associated with a very high rate of non-recovery of renal function. ${ }^{[6]}$

It is increasingly apparent that those diseases traditionally ascribed as risk factors for AKI (e.g. heart disease, hypertension and diabetes) in the developed world are increasing in prevalence in developing countries. Current estimates suggest that approximately 25\% of South Africa (SA)'s population suffer from hypertension. ${ }^{[7]}$ Similarly, diabetes is believed to affect 9.4 million people in Africa, with a prevalence of diabetic nephropathy reaching $14-16 \%$ in SA, $9 \%$ in Sudan and $6.1 \%$ in Ethiopia. ${ }^{[7]}$ Previous studies of AKI in subSaharan Africa have highlighted obstetric complications to be an important cause of AKI. In SA, pregnancy-related disorders (most 
notably septic abortion and pre-eclampsia) are responsible for $15 \%$ of all cases of AKI requiring dialysis. ${ }^{[8]}$ Although data regarding the nature of AKI in Ethiopia are limited, pregnancy-related disorders are similarly implicated in AKI in an Ethiopian population. ${ }^{[9]}$

In patients undergoing major surgery, postoperative AKI occurs in $33 \%$ of patients $>65$ years old. ${ }^{[10]}$ Pre-existing CKD is known to be a potent predictor of postoperative AKI and is associated with failure of recovery of renal function. ${ }^{[11]}$ There are well-defined patient cohorts who are at high risk and would benefit from early identification and monitoring with institution of appropriate interventions to avoid development of AKI. The aim of the present study was to assess current clinical practice in terms of identification of patterns of AKI risk factor assessment, and relate this information to monitoring of renal function in patients admitted to three teaching hospitals in Ethiopia.

\section{Methods}

Data were collected in three teaching hospitals across Ethiopia (identified in the tables below as: $\mathrm{A}=$ Tikur Anebassa Hospital in Addis Ababa; $\mathrm{B}=$ Gondar University Hospital; $\mathrm{C}=$ Awassa University Hospital). The Tikur Anebassa is a 700-bed hospital with 110 medical beds. It is a tertiary referral centre for the whole of Ethiopia and has 2 nephrologists. The hospitals at Gondar and Awassa are both teaching hospitals in a rural setting with no specialist nephrology services. The former is a 400-bed (120 medical beds) referral hospital for NorthWest Ethiopia, serving a population of 5 million people, and the latter a 350-bed (52 medical beds) referral hospital for the Southern Nations, Nationalities and Peoples Region (SNNPR) of Ethiopia with a population of 16 million.

Cross-sectional data were gathered for patients in the medical, surgical and obstetrics and gynaecology wards. Data were collected on all in-patients from their medical records over a one-week period at each centre during July 2011. Patients were excluded if they were under the age of 16 years, if the patient records were unavailable, or if they had been admitted within 12 hours of the time of data collection (where not all patients would have been seen by a doctor.)

\section{Risk factor monitoring}

Analysis of risk factor recognition was based on two types of risk factor: comorbidities and nephrotoxic medications. Evidence was sought in documentation for specific comorbidities known to be associated with increased risk of AKI: heart disease, hypertension, diabetes mellitus, HIV, cerebrovascular disease or pre-existing CKD. Nephrotoxic medications were defined as angiotensin-converting enzyme (ACE) inhibitors, nonsteroidal anti-inflammatory drugs
(NSAIDs) or aminoglycoside antibiotics (monitoring of aminoglycoside levels was available at each centre). Lack of documentation for comorbidities may reflect either a genuine lack of comorbidities, or a lack of enquiry and documentation; therefore, additional data were sought from patient records via systematic enquiry of patients' past medical history.

\section{Investigations and monitoring}

Whether renal function was measured (measurement of serum creatinine, blood urea nitrogen or both), whether the results were abnormal, and whether measurement was repeated, was documented for each patient. In addition, it was noted whether urine output, urine analysis by dipstick, or blood pressure were recorded and whether these investigations were repeated.

\section{Statistical analysis}

Data are presented as mean \pm standard deviation (range). Comparison between groups was performed by chi-square test, and $p<0.05$ taken to represent statistical significance.

\section{Results}

\section{Patient characteristics and underlying diagnoses}

Across the 3 centres, 366 patients were recruited. Patient demographics are shown in Table 1 . The only significant difference between patient groups was the expected younger age of those in Obstetrics and Gynaecology (O\&G) wards. Similarly, duration of hospital stay was no different between each centre for each clinical setting, suggesting a similar level of clinical dependency. Active clinical problems are summarised in Figs 1 - 3. The most common reason for admission to a medical ward was infection (Fig. 1 - 3). For those medical patients labelled with 'infectious' cause for admission, the most common diagnoses were HIV and TB. At each centre, the percentage of the infections attributed to HIV was $17 \%$ (Centre A), 52\% (Centre B) and 39\% (Centre C); and for TB, 20\% (Centre A), 64\% (Centre B) and 33\% (Centre $C$ ). Almost all patients in surgical wards underwent surgery during their admission (Centre A - 90\%, Centre B - 94\%, Centre $\mathrm{C}-$ data not available). The majority of surgical procedures were performed electively (hospital A - 67\%, hospital B - 55\%, hospital C - data not available). The proportion of patients from the O\&G wards categorised as obstetric cases varied between the three centres (Centre A - 53\%, Centre B - 62\%, Centre C - 82.5\%).

\section{Investigations performed}

Table 2 shows the percentage of patients in whom renal function was monitored. There was significant variation between different centres,

Table 1. Summary of patient age, sex and duration of hospital stay

\begin{tabular}{lllll}
\hline Ward (Centre) & Number of patients & Age (years) & \% male & Average length of stay (days) \\
\hline Medicine (A) & 45 & $39.7 \pm 18$ & 49 & $16.9 \pm 14$ \\
Surgery (A) & 51 & $43.5 \pm 14$ & 53 & $8.5 \pm 10$ \\
O\&G (A) & 34 & $33.5 \pm 13$ & 0 & $8.2 \pm 8$ \\
Medicine (B) & 52 & $38.9 \pm 18$ & 52 & $16.4 \pm 19$ \\
Surgery (B) & 53 & $37.2 \pm 14$ & 75 & $13.2 \pm 11$ \\
O\&G (B) & 42 & $29.1 \pm 9$ & 0 & $14.7 \pm 17$ \\
Medicine (C) & 33 & $40.6 \pm 20$ & 67 & $6.4 \pm 6$ \\
Surgery (C) & 33 & $38.1 \pm 20$ & 58 & $4.7 \pm 4.8$ \\
O\&G (C) & 23 & $26.6 \pm 6$ & 0 & $5.3 \pm 6$ \\
The data are shown independently for each of the hospitals (A, B and C) and for each ward setting (Medicine, Surgery and O\&G). Data are presented as mean \pm standard deviation.
\end{tabular}




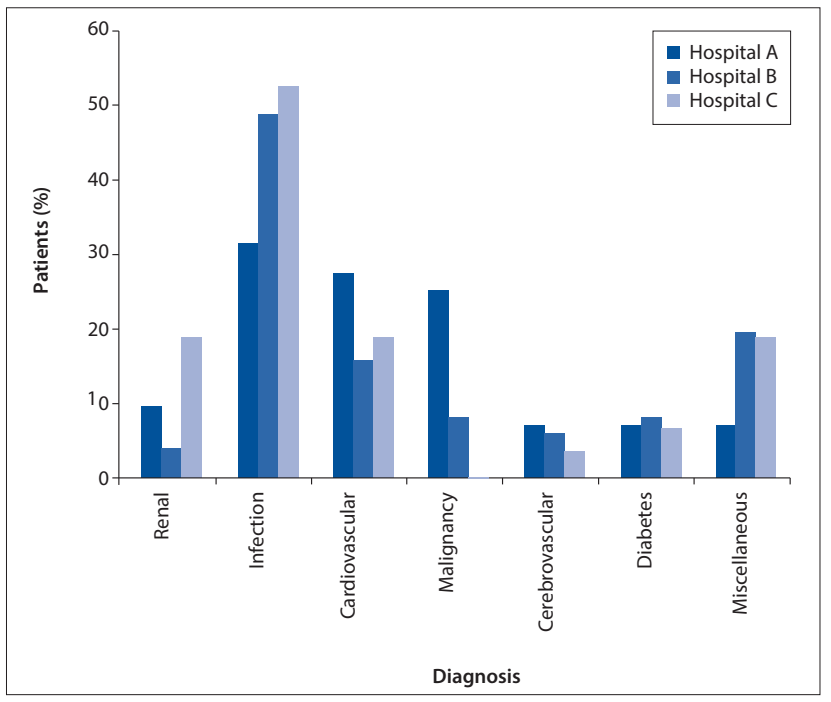

Fig. 1. Working clinical diagnoses of patients on medical wards at the each of the 3 centres.

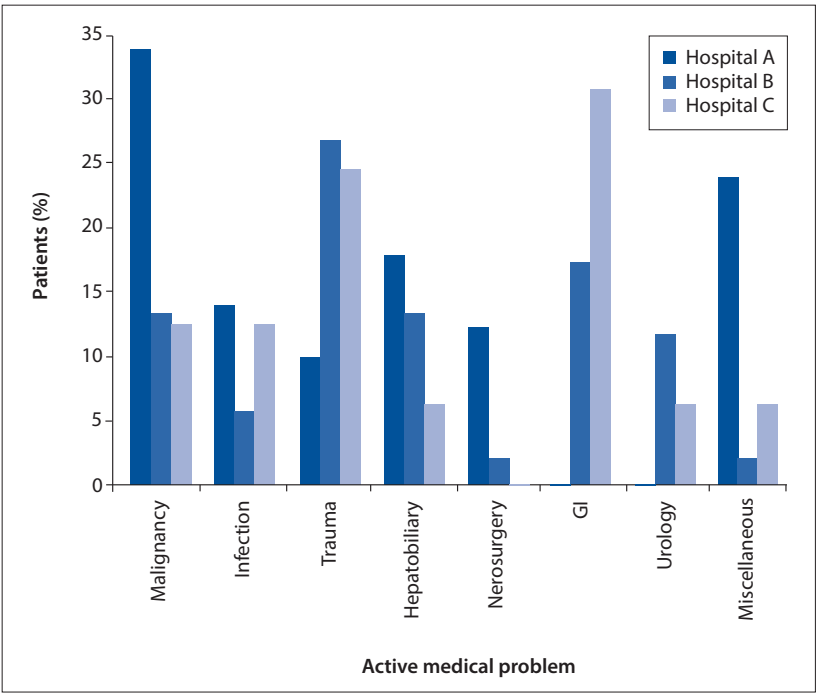

Fig. 2. Working clinical diagnoses of patients admitted to surgical wards at each of the 3 centres.

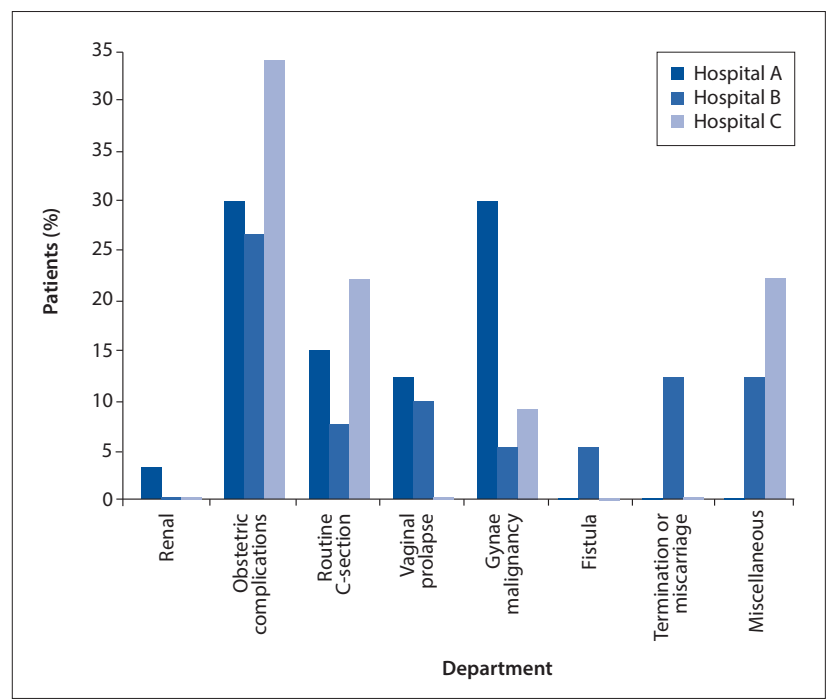

Fig. 3. Active clinical problems in $O \mho G$ patients in each of the 3 centres. and across the different specialities within each centre. Patients were significantly more likely to have had their renal function checked if they were at the hospital with specialist nephrology services (Centre A - 75\%, Centre B - 31\%, Centre C - 30\%) $(p<0.001)$. In each centre, a statistically higher proportion of patients admitted to a medical ward had renal function measurements (Centres $\mathrm{A}$ and $\mathrm{B} p<0.0001$ and Centre $\mathrm{C} p=0.002$ ).

The proportion of patients who had urine output and/or urinalysis performed was low in all centres and in all departments. There was no difference between each centre, nor across the departments at each centre, in the probability either of urine output to have been measured or of urinalysis to have been performed.

Blood pressure measurement was recorded for the majority of patients in all centres and all wards, with the exception of the surgical ward of one of the centres.

\section{Recognition of risk factors}

\section{Recording of AKI risk-associated comorbidities}

Evidence that systematic enquiry of a patient's past medical history was most probably undertaken in medical wards in each centre, suggest a lack of awareness of the need for such a risk assessment outside the context of the general medical ward (Fig. 4). For this reason, patients in medical wards were more likely to have documentation of AKI risk-associated comorbidities in their clinical records (Table 3).

\section{Nephrotoxic agents}

The percentages of patients prescribed one or more nephrotoxic agents in each centre and across all wards is shown in Fig. 5. ACE inhibitors were most likely to have been prescribed in medical wards (ACE inhibitor prescription: medical patients in Centres A, B and C: $31 \%, 4 \%, 12 \%$; surgical patients in Centres A, B and C: $4 \%, 0 \%$, $0 \%$; O\&G patients in Centres A, B and C: $0 \%, 0 \%, 4 \%$; respectively). Prescription of aminoglycosides and NSAIDs was highest in surgical wards (aminoglycoside prescription: surgical patients in Centres A, B and C: $6 \%, 17 \%, 39 \%$; medical patients in Centres A, B and C: $18 \%, 9 \%, 0 \%$; and O\&G patients in Centres A, B and C: $6 \%, 12 \%$, 6\%; respectively. NSAID prescription: surgical patients in Centres A, B and C: $27 \%, 41 \%, 58 \%$; medical patients in Centres A, B and C: $13 \%, 13 \%, 21 \%$; and O\&G patients in Centres A, B and C: $32 \%, 16 \%$, $44 \%$; respectively). In the surgical wards, $5.8 \%, 5.6 \%$ and $33.3 \%$ of all patients were on a combination of aminoglycosides and NSAIDs, at Centres A, B and C respectively.

\section{Relationship between risk factor assessment and monitoring of renal function}

For the entire patient cohort from the 3 centres, patients who had documentation of $\geq 1$ AKI risk factor-associated comorbidities were significantly more likely to have their renal function measured than the group for which there was no such documentation ( $65 \%$ v. $31 \%$ $p<0.0001$ by chi-square test). This relationship remained significant when calculated for each of the individual hospitals: Centre A $89 \%$ v. $60 \%, p=0.0004$; Centre B $54 \%$ v. $16 \%, p<0.001$; Centre C $43 \%$ v. $22 \%, p=0.038$.

The prescription of nephrotoxic agents did not influence the frequency of measurement of renal function, with the proportion of those patients who had their renal function measured being no different between the cohort prescribed at least one nephrotoxic agent and that which received no nephrotoxic agent (Table 4). For all departments and each centre (except Surgery, Centre A), the relationship between prescription of nephrotoxic agents and measurement of renal function was not significant. In Centre A 
Surgery, patients who were not prescribed nephrotoxic agents were, surprisingly, statistically more likely to have their renal function measured $(p=0.04)$.

\section{Discussion}

One key objective in reducing the incidence of AKI is the assessment and management of factors that place patients at risk of the condition. Established risk factors include comorbidities such as cardiovascular risk factors (hypertension, diabetes mellitus), cardiovascular disease and severe infections such as HIV. The aim of this study was to explore patterns of AKI risk factor recognition and the relationship with subsequent renal monitoring across 3 different teaching hospital centres in Ethiopia, encompassing 9 different in-patient ward settings.

The first key learning point derived from the data relates to the detection and documentation rates of AKI risk factors among departments. In general, AKI risk factor assessment and documentation was highest in the hospital with specialist nephrology services; across all 3 centres, AKI risk factor assessment was highest in the medical wards. There was, however, marked variation in the extent to which doctors actively sought to identify the presence of such comorbidities. While some wards aimed to ensure complete documentation of past medical history and comorbidities, some failed to enquire about relevant conditions in as much as $80 \%$ of patients. Failure to collect this information resulted in incomplete medical records, and represented a lost opportunity for identifying patients at high risk of developing AKI.

Failure to record all clinical details in inpatient medical notes is recognised as a significant problem worldwide. Contributing factors include limited time available for clerking new patients, and numerous demands placed on junior doctors responsible for ensuring that wards run smoothly. ${ }^{[12]}$ One way to address these issues is to produce standardised preprinted medical admission sheets for junior doctors to use as prompting tools. Many studies have provided

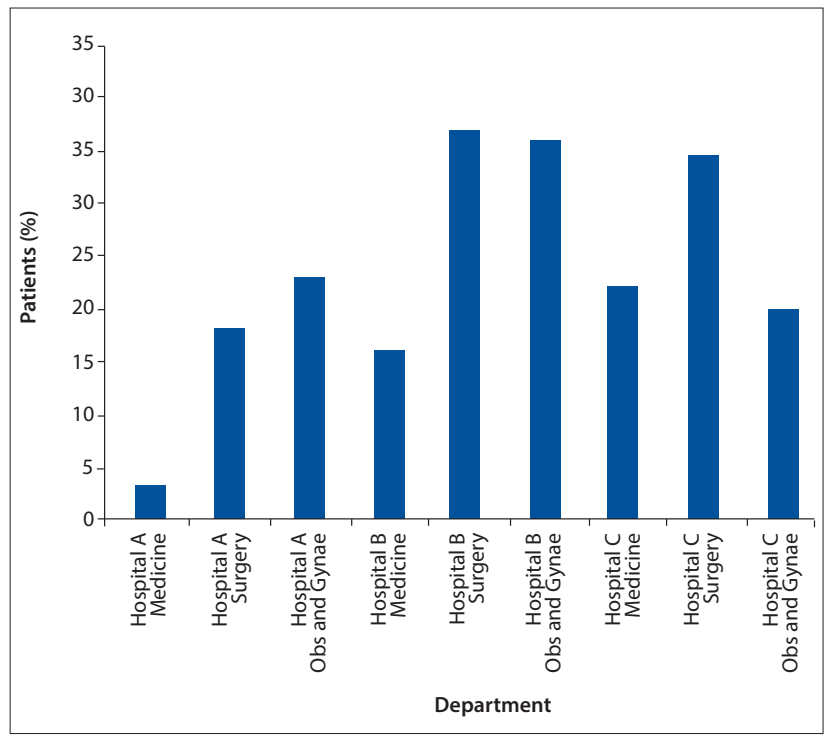

Fig. 4. Percentage of patients in each department for whom there was no documented evidence of a systematic enquiry of past medical history in each of the 3 centres.

Table 2. Percentage of patients in whom renal function was assessed by each of the parameters indicated, in each centre and in each ward setting

\begin{tabular}{lllll}
\hline Ward (Centre) & Renal function (\%) & Urine output (\%) & Dipstick (\%) & BP (\%) \\
\hline Medicine (A) $n=45$ & 100 & 20 & 30 & 98 \\
Surgery (A) $n=41$ & 74 & 16 & 25 & 98 \\
O\&G (A) $n=34$ & 41 & 6 & 32 & 100 \\
Medicine (B) $n=52$ & 63 & 17 & 50 & 94 \\
Surgery (B) $n=53$ & 15 & 5 & 19 & 57 \\
O\&G (B) $n=42$ & 9 & 0 & 31 & 83 \\
Medicine (C) $n=33$ & 51 & 6 & 57 & 61 \\
Surgery (C) $n=33$ & 12 & 0 & 27 & 88 \\
O\&G (C) $n=23$ & 26 & 4 & 52 & 100
\end{tabular}

Table 3. Percentage of patients with 0 - 4 documented AKI risk-associated comorbidities in each centre and in each ward setting

\begin{tabular}{|c|c|c|c|c|c|c|}
\hline Ward (Centre) & $\begin{array}{l}0 \\
\text { comorbidity }\end{array}$ & $\begin{array}{l}1 \\
\text { comorbidity }\end{array}$ & $\begin{array}{l}2 \\
\text { comorbidities }\end{array}$ & $\begin{array}{l}3 \\
\text { comorbidities }\end{array}$ & $\begin{array}{l}4 \\
\text { comorbidities }\end{array}$ & $\begin{array}{l}\text { Average number of } \\
\text { comorbidities }\end{array}$ \\
\hline Medicine (A) & 7 & 51 & 29 & 11 & 2 & 1.5 \\
\hline Surgery (A) & 69 & 25 & 6 & 0 & 0 & 0.4 \\
\hline O\&G (A) & 88 & 9 & 3 & 0 & 0 & 0.1 \\
\hline Medicine (B) & 21 & 38 & 37 & 4 & 0 & 1.2 \\
\hline Surgery (B) & 83 & 17 & 0 & 0 & 0 & 0.2 \\
\hline O\&G (B) & 86 & 14 & 0 & 0 & 0 & 0.1 \\
\hline Medicine (C) & 9 & 61 & 27 & 3 & 0 & 1.2 \\
\hline Surgery (C) & 88 & 12 & 0 & 0 & 0 & 0.1 \\
\hline O\&G (C) & 96 & 4 & 0 & 0 & 0 & 0 \\
\hline
\end{tabular}


evidence that preprinted pro formas for patient admission are effective in overcoming problems with incomplete medical records. Not only do these forms increase data capture, ${ }^{[12-14]}$ but they also make the process of gathering clinical information at the point of admission more efficient and less time-consuming. These findings have been replicated in hospitals across the world, including in Africa. ${ }^{[15]}$

Following any risk assessment, it is also imperative that a diagnosis of any 'comorbidity' should prompt doctors to conduct renal function testing and monitoring. The second key message from the data is that documentation of AKI risk-associated comorbidities was associated with a greater awareness of the importance of measuring renal function, which suggests that Ethiopian doctors appreciate the importance of comorbidities as renal risk factors and the need for renal monitoring in affected patients. This statement requires qualification, however, as measurement of renal function was highest in those specialities (i.e. medicine) with the highest rate of documentation and enquiry of risk assessment, which implies that awareness of AKI outside the context of medical admissions is generally low.

While the patient's clinical history provides one important risk assessment tool for AKI, it is also important to consider the risks associated with the treatment instituted while the

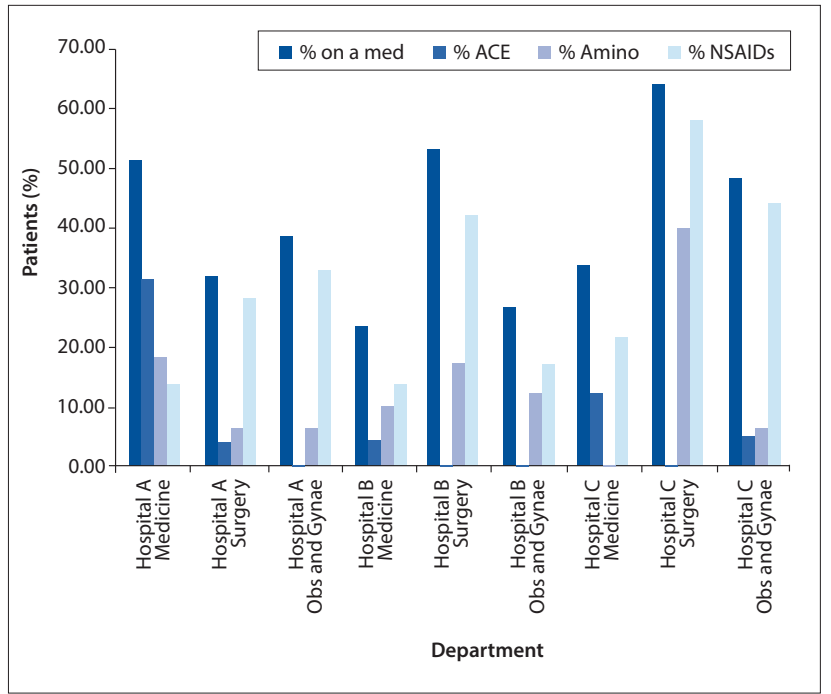

Fig. 5. Percentage of patients prescribed 'nephrotoxic' pharmacological agents according to department in each of the 3 centres. patient is in hospital. A study conducted in an Ethiopian hospital has highlighted the burden of renal injury associated with the prescription of nephrotoxic drugs, with up to $10 \%$ of AKI directly ascribed to the use of nephrotoxic agents. ${ }^{[9]}$ Our data, comparing the prescribing patterns in each of the different departments, revealed great variability in the frequency of prescription of nephrotoxic medications. ACE inhibitors were most commonly prescribed in medical wards, probably reflecting an older patient cohort and presence of cardiovascular disease. Conversely, NSAIDs and aminoglycosides were more frequently prescribed among surgical patients for management of postoperative pain relief and prophylaxis of postoperative infection, respectively. In general, patients treated with nephrotoxic agents were not identified as an at-risk population, and therefore were not monitored for evidence of deteriorating renal function; this constitutes a lost opportunity for identifying and managing patients at high risk of AKI. Although the risk of developing nephrotoxicity following NSAID use is reported to be relatively low, the extensive prescription of NSAIDs for analgesia, when alternative agents are available, places many patients at unnecessary risk. ${ }^{[16,17]}$ It would be appropriate to review the choice of first-line agents in certain settings, especially for patients with other predisposing comorbidities. Undertaking careful monitoring of renal function in patients taking nephrotoxic medications would ensure early recognition of renal toxicity. If the drug is necessary, dosing intervals could be altered as appropriate for the degree of renal impairment.

In summary: We have demonstrated that the presence of important risk factors for the development of AKI frequently goes unrecognised. This situation is not unique to Ethiopia. The recent National Confidential Enquiry into Perioperative Deaths (NCEPOD) report on AKI in the UK suggested that 29\% of patients did not have adequate assessment or documentation of the most important risk factors for AKI, and that, as in the current study, medication and comorbidity were among the most common risk factors not assessed. ${ }^{[5]}$ In a country such as Ethiopia where access to dialysis is severely restricted, early identification of AKI and pre-empting the need for renal replacement therapy is of particular importance, and is likely to directly affect patient mortality. Identification of high-risk patients would be improved by providing education about the key risk factors associated with the development of AKI, and instituting protocols and systems whereby subgroups of high-risk patients might be targeted for monitoring. Understanding current practice is the key first step in the design of any future strategy for minimising risk. With the

Table 4. Relationship between prescription of nephrotoxic agents and measurement of renal function

\begin{tabular}{|c|c|c|c|}
\hline Ward (Centre) & $\begin{array}{l}\text { Percentage of patients prescribed a nephrotoxic } \\
\text { drug who had renal function checked }\end{array}$ & $\begin{array}{l}\text { Percentage of patients not prescribed a } \\
\text { nephrotoxic drug who had renal function checked }\end{array}$ & $p$-value \\
\hline Medicine (A) & 100 & 100 & $\mathrm{n} / \mathrm{s}$ \\
\hline Surgery (A) & 56 & 83 & 0.04 \\
\hline O\&G (A) & 39 & 43 & $\mathrm{n} / \mathrm{s}$ \\
\hline Medicine (B) & 75 & 60 & $\mathrm{n} / \mathrm{s}$ \\
\hline Surgery (B) & 18 & 12 & $\mathrm{n} / \mathrm{s}$ \\
\hline O\&G (B) & 9 & 10 & $\mathrm{n} / \mathrm{s}$ \\
\hline Medicine (C) & 46 & 50 & $\mathrm{n} / \mathrm{s}$ \\
\hline Surgery (C) & 14 & 8 & $\mathrm{n} / \mathrm{s}$ \\
\hline O\&G (C) & 27 & 25 & $\mathrm{n} / \mathrm{s}$ \\
\hline
\end{tabular}


data presented, and with the support of the International Society of Nephrology's Sister Centre Programme, we are well placed to develop a platform for future learning that will disseminate key messages aimed at modifying medical behaviour. The provision of locally applicable guidelines, which can drive a self-sustaining cycle of audit, should ultimately have a positive effect on the prevalence of AKI in Ethiopia.

Acknowledgments. This work was supported by the International Society of Nephrology's Sister Centre Programme, which was established in 2010. Travel costs were supported by the award of a Baxter Clinical Evidence Council Research Grant.

\section{References}

1. Kaballo BG, Khogali MS, Khalifa EH, Khaiii EA, Ei-Hassan AM, Abu-Aisha H. Patterns of "severe acute renal failure" in a referral center in Sudan: excluding intensive care and major surgery patients. Saudi J Kidney Dis Transpl 2007;18(2):220-225.

2. Arogundade FA, Sanusi AA, Okunola OO, Soyinka FO, Ojo OE, Akinsola A. Acute renal failure (ARF) Arogundade FA, Sanusi AA, Okunola OO, Soyinka FO, Ojo OE, Akinsola A. Acute renal failure (ARF) 3. Cerda J, Lameire N, Eggers P, et al. Epidemiology of acute kidney injury. Clin J Am Soc Nephrol 2008;3(3):881-886. [http://dx.doi.org/10.2215/CJN.04961107]

2008; 3(3):881-886. [http://dx.doi.org/10.2215/CJN.04961107]
Okusa MD, Chertow GM, Portilla D. The nexus of acute kidney injury, chronic kidney disease, and World 4. Okusa MD, Chertow GM, Portilla D. The nexus of acute kidney injury, chronic kidney disease, and World
Kidney Day 2009. Clin J Am Soc Nephrol 2009;4(3):520-522. [http://dx.doi.org/10.2215/CJN.06711208]
5. NCEPOD. National Confidential Enquiry into Patient Outcome and Death [NCEPOD] Report on Acute Kidney Injury: Adding Insult to Injury. NCEPOD, London:2009.

6. Hsu CY, Chet ow GM, MeCulloch CE, Fin D, Ordone JD, Go AS. Nonrecovery of kidney function and death after acute on chronic renal failure. Clin J Am Soc Nephrol 2009;4(5):891-898. [http://

dx.doi.org/10.2215/CJN.05571008]
Naicker S. End-stage renal disease in sub-Saharan Africa. Ethn Dis 2009;19(S1):13-15.

7. Naicker S. End-stage renal disease in sub-Saharan Africa. Ethn Dis 2009;19(S1):13-15. Africa. Ren Fail 1995;17(2):147-153.

9. Zewdu W. Acute renal failure in Addis Abeba, Ethiopia: a prospective study of 136 patients. Ethiop Med J 1994;32(2):79-87.

10. Gong Y, Zhang F, Ding F, Gu Y. Elderly patients with acute kidney injury (AKI): clinical features and risk factors for mortality. Arch Gerontol Geriatr 2012;54(2):47-51. [http://dx.doi.org/10.1016/j. archger.2011.05.011]

11. Wu VC, Huang TM, Lai CF, et al. Acute-on-chronic kidney injury at hospital discharge is associated with long-term dialysis and mortality. Kidney Int 2011;80(11):1222-1230. [http://dx.doi.org/10.1038/ ki.2011.259]

12. Diver AJ, Craig BF. Admission proforma significantly improves the medical record. Scott Med J 2005;50(3):101-102.

13. Belmin J, de la Fourniere F, Bellot P, et al. Quality of the information collected during admission to a hospital geriatric service: importance of a structured medical record. Presse Med 1998;27(30):15191522.

14. Goodyear HM, Lloyd BW. Can admission notes be improved by using preprinted assessment sheets? Qual Health Care 1995;4(3):190-193.

15. Mwakyusa S, Wamae A, Wasunna A, et al. Implementation of a structured paediatric admission record for district hospitals in Kenya - results of a pilot study. BMC Int Health Hum Rights 2006;6:9. [http:// dx.doi.org/10.1186/1472-698X-6-9]

16. Unsworth J, Sturman S, Lunec J, Blake DR. Renal impairment associated with non-steroidal antiinflammatory drugs. Ann Rheum Dis 1987;46(3):233-236.

17. Whelton A. Nephrotoxicity of nonsteroidal anti-inflammatory drugs: physiologic foundations and clinical implications. Am J Med 1999;106(5B):13S-24S.

Accepted 11 March 2013.

This month in the $S A M J . .$.

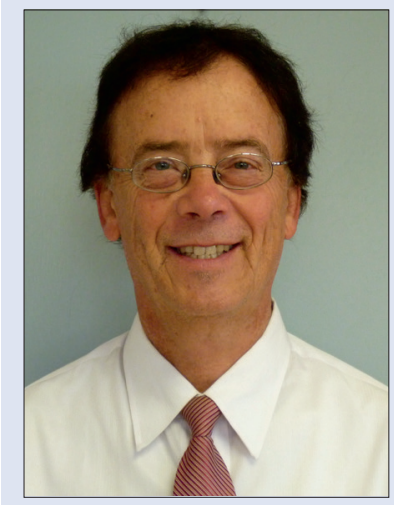

Paul Potter ${ }^{*},{ }^{+}$is the director of the Allergology, Diagnostic and Clinical Research Unit of the Lung Institute at the University of Cape Town and head of Allergology in the Department of Medicine at Groote Schuur Hospital. He is a past member of the Board of Directors of the World Allergy Organisation and served on their council on Specialisation and Training in Allergology. He holds a doctorate in molecular biology and allergy for his work on the human $\beta 2$ receptor. He has directed his research efforts towards developing the field of allergology in South Africa, focusing on indigenous and local characterisation, allergen immunotherapy, complement deficiency diseases and latex allergy. In 1999, he founded the Allergy Diagnostic and Clinical Research Unit at the Lung Institute as a centre of excellence that specialises in clinical and laboratory diagnosis and management of allergic patients and also provides training facilities for diplomates and fellows specialising in allergology.

'Potter P. Latex allergy: Plight, rights and fights. SAfr Med 2013;103(6):369-370. [http://dx.doi.org/10.7196/SAMI.7015]

† Green R, Hockman M, Friedman R, et al. Chronic Rhinitis in South Africa - Update 2013. S Afr Med J 2013;103(6):419-422. [http//dx.doi.org/10.7196/SAMI.6972]

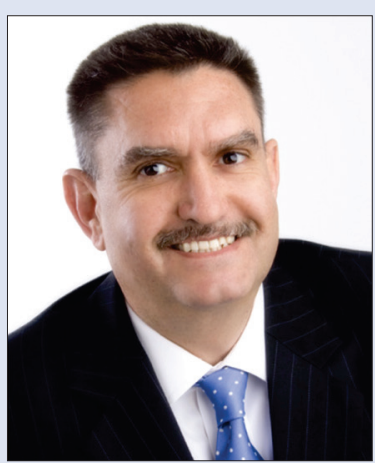

Robin J Green ${ }^{*},{ }^{+}$is director of Paediatric Services and Paediatric Pulmonology within the Paediatric Intensive Care and Allergy Services at the Steve Biko Academic Hospital, Pretoria. He is a professor in the Department of Paediatrics and Child Health within the School of Medicine at the University of Pretoria. He holds an MB $\mathrm{BCh}$ and $\mathrm{PhD}$, is a fellow of the Royal College of Physicians and president of the College of Paediatricians of South Africa. He is also chairman of the Allergy Society of South Africa. The National Research Foundation rates him as an established researcher, specialising in paediatric pulmonology. His research interests concern asthma and its associated co-morbidities with a focus on asthma control. He believes that if our aim is to ensure that all asthmatics lead a normal life we still have a long way to go.

* Green R, Hockman M, Friedman R, et al. Chronic Rhinitis in South Africa - Update 2013. S Afr Med J 2013:103(6):419-422. [http://dx.doi.org/10.7196/SAMI.6972] † Risenga SM, Shivambu GP, Rakgole MP, Makwela LM, Maligavhada NJ, Green R. Latex allergy in Healthcare workers at a Teaching Hospital in Limpopo, South Africa. S Afr Med J 2013:103(6):390-394. [http:///dx.doi org/10.7196//AMI.6011] 